\title{
Spectral beam combining of diode lasers with high efficiency
}

Müller, André; Vijayakumar, Deepak; Jensen, Ole Bjarlin; Hasler, Karl-Heinz ; Sumpf, Bernd ; Erbert, Götz ; Andersen, Peter E.; Petersen, Paul Michael

Published in:

Lasers, Sources, and Related Photonic Devices Technical Digest

Publication date:

2012

Document Version

Publisher's PDF, also known as Version of record

Link back to DTU Orbit

Citation (APA):

Müller, A., Vijayakumar, D., Jensen, O. B., Hasler, K-H., Sumpf, B., Erbert, G., Andersen, P. E., \& Petersen, P. M. (2012). Spectral beam combining of diode lasers with high efficiency. In Lasers, Sources, and Related Photonic Devices Technical Digest (pp. AM4A.10). Optical Society of America.

\section{General rights}

Copyright and moral rights for the publications made accessible in the public portal are retained by the authors and/or other copyright owners and it is a condition of accessing publications that users recognise and abide by the legal requirements associated with these rights.

- Users may download and print one copy of any publication from the public portal for the purpose of private study or research.

- You may not further distribute the material or use it for any profit-making activity or commercial gain

- You may freely distribute the URL identifying the publication in the public portal 


\title{
Spectral beam combining of diode lasers with high efficiency
}

\author{
André Müller, ${ }^{1, *}$ Deepak Vijayakumar, ${ }^{1}$ Ole Bjarlin Jensen, ${ }^{1}$ Karl-Heinz Hasler, ${ }^{2}$ \\ Bernd Sumpf, ${ }^{2}$ Götz Erbert, ${ }^{2}$ Peter E. Andersen, ${ }^{1}$ and Paul Michael Petersen ${ }^{1}$ \\ ${ }^{I}$ DTU Fotonik, Department of Photonics Engineering, Technical University of Denmark, Frederiksborgvej 399, 4000 Roskilde, Denmark \\ ${ }^{2}$ Ferdinand-Braun-Institut, Leibniz-Institut für Höchstfrequenztechnik, Gustav-Kirchhoff-Straße 4, 12489 Berlin, Germany \\ amul@fotonik.dtu.dk
}

\begin{abstract}
Based on spectral beam combining we obtain $16 \mathrm{~W}$ of output power, combining two $1063 \mathrm{~nm}$ DBR-tapered diode lasers. The spectral separation within the combined beam can be used for subsequent sum-frequency generation.

OCIS codes: (140.2020) Diode lasers; (140.3298) Laser beam combining; (190.2620) Harmonic generation and mixing
\end{abstract}

\section{Introduction}

Diode lasers are by far the most efficient light sources but suffer from heat effects that can cause their degradation especially in the high-power CW regime. A promising straight-forward approach to increase the output power of moderately operated diode laser modules without affecting the beam quality is spectral beam combining. This technique describes the combining of two or multiple lasers using diffractive optical elements like diffraction gratings or volume Bragg gratings. In both cases power scaling can be achieved with combining efficiencies > $90 \%$.

Spectral beam combining requires the lasers to have slightly different wavelengths. Experiments based on diffraction gratings have shown spectral separations between $2.5 \mathrm{~nm}$ to $5 \mathrm{~nm}$. When using similar diode lasers this separation needs to be decreased. This can be explained by the tunability of diode lasers. To some extent the emission wavelengths of diode lasers can be tuned by changing the laser temperature. However, shifting the emission wavelength over several nanometers as mentioned above would require large changes in temperature that could cause heat effects limiting the performance. One way around this issue is to increase the source-to-grating distances, prohibiting combining systems with low footprints. A more compact alternative is to use diode laser arrays [1]. Unfortunately, lower combining efficiencies achieved in this approach might be a result from the reduced flexibility in terms of optical alignments and adjustments of the laser parameters. Additionally, diode laser arrays have the disadvantage of mounting induced smiles degrading the beam quality of the combined beam. Spectral beam combining of single emitters instead shows the resulting beam quality to match the values of the individual lasers. This indicates the general potential of achieving near diffraction limited high-power diode laser systems by choosing the right lasers. In this context volume Bragg gratings have been shown to be a suitable alternative for combining lasers with wavelengths shifted only around $0.5 \mathrm{~nm}$. These gratings are written in photo-thermo-refractive glass and therefore offer excellent thermo-mechanical properties, high transmittance in the near-infrared and diffraction efficiencies close to unity [2]. The spectral spacing between maximum and zero diffraction simply depends on the spatial frequency of the grating.

We present spectral beam combining of $1063 \mathrm{~nm}$ DBR-tapered diode lasers. These lasers are capable of emitting up to $12 \mathrm{~W}$ with nearly diffraction limited beam qualities [3]. Using a volume Bragg grating we achieve combining efficiencies between $93 \%-96 \%$. The maximum output power is $16 \mathrm{~W}$ with beam propagation parameters of 1.8 in the fast axis and 3.3 in the slow axis. Based on these results future systems could offer several tens of Watts with single, near diffraction limited output beams. Furthermore, the spectral separation of two combined lasers offers the opportunity for subsequent sum-Frequency mixing. In this context we already achieved more than $2 \mathrm{~W}$ of green light and we are planning to show further results.

\section{Experimental setup}

Fig. 1 shows a simplified illustration of our setup for spectral beam combining of two DBR-tapered diode lasers. Both lasers (1) have a total length of $6 \mathrm{~mm}$ and are mounted p-side up on a $\mathrm{CuW}$ heat spreader, mounted on a $25 \times 25 \mathrm{~mm}^{2}$ conduction cooled package mount for efficient cooling. The injection currents to the ridge waveguide and to the tapered amplifier of the diode lasers are controlled individually.

The elliptic beams emitted by both lasers are collimated using AR-coated aspheric lenses (2) with focal lengths of $f=3.1 \mathrm{~mm}$ and numerical apertures of $N A=0.68$, and cylindrical lenses (3) with focal lengths of $f=15 \mathrm{~mm}$. This highly reduces the feedback from the lens surfaces and helps to compensate the astigmatism of the diode lasers. The resulting beam has a beam diameter of about $2 \mathrm{~mm}$.

The grating (4) used for spectral beam combining is a reflecting volume Bragg grating (OptiGrate) with the dimensions $\mathrm{L} \times \mathrm{W} \times \mathrm{H}=(3.4 \times 10 \times 10) \mathrm{mm}^{3}$. The specified average diffraction efficiency is $99.2 \%$ at $1062 \mathrm{~nm}$ 
with a spectral selectivity of $0.3 \mathrm{~nm}(\mathrm{FWHM})$. For spectral beam combining the laser not fulfilling the Bragg condition simply transmits the grating. The beam of the second laser fulfilling the condition hits the grating from the other side using regular mirrors (5) and is diffracted into the same direction as the transmitted beam. The required Bragg condition for optimum spectral beam combining is obtained by adjusting the angle of incidence on the grating and the wavelength of the beam.

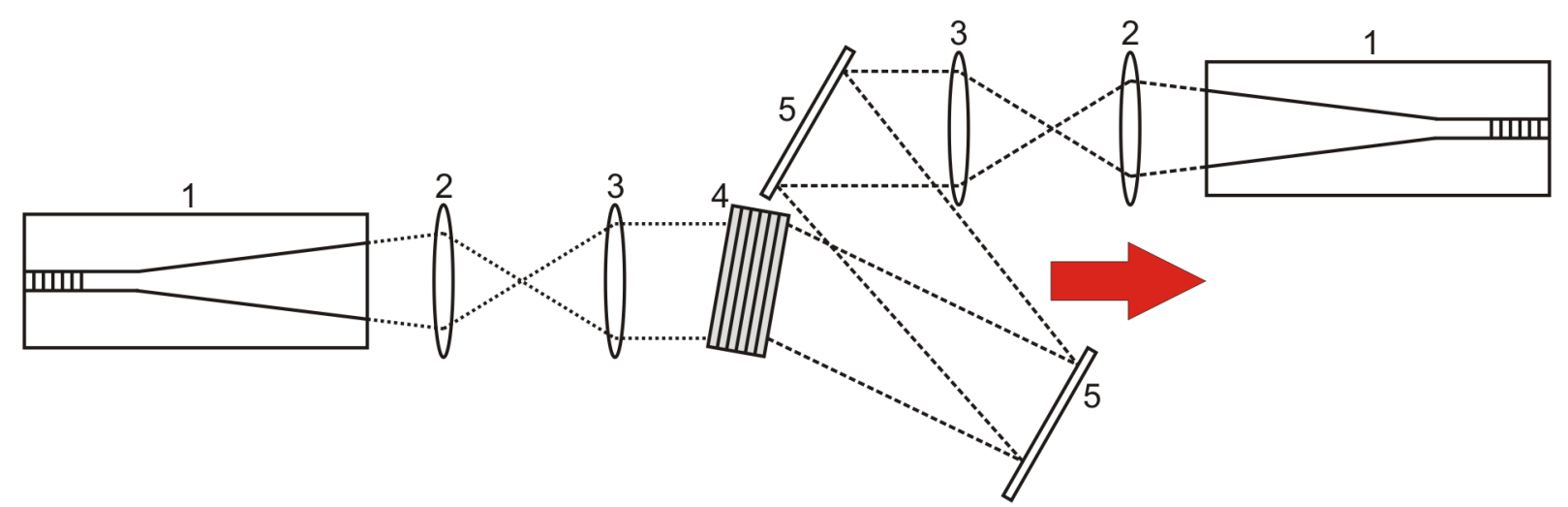

Fig. 1. Simplified illustration of the setup for spectral beam combining of two diode lasers. The individual beam paths are illustrated by dots (transmitted laser) and dashes (diffracted laser). The arrow is pointing into the direction of propagation of the combined beam.

\section{Results}

As mentioned above, the injection currents are controlled individually. During the experiments the ridge waveguides are operated at $300 \mathrm{~mA}$. The maximum current to the tapered amplifiers is $14 \mathrm{~A}$ resulting in laser emissions of $8.46 \mathrm{~W}(0.71 \mathrm{~W} / \mathrm{A})$ from the laser diffracted by the grating and $8.92 \mathrm{~W}(0.74 \mathrm{~W} / \mathrm{A})$ from the transmitted laser at temperatures of $20{ }^{\circ} \mathrm{C}$ (Fig. 2). The lasers do not show any signs of thermal roll over. The corresponding beam propagation parameters $\left(M^{2}{ }_{1 / e^{2}}\right)$ are measured to be 1.4 (fast axis) and 3.2 (slow axis) for the diffracted laser and 1.5 (fast axis) and 2.1 (slow axis) for the transmitted laser. The emission spectra shown by the inset of Fig. 2 are located around $1063 \mathrm{~nm}$. The spectral widths are measured to be $6 \mathrm{pm}$ limited by the spectral resolution of the optical spectrum analyzer (Advantest Q8347). The side mode suppression is $>25 \mathrm{~dB}$. Adjusting the wavelengths of the diode lasers for spectral beam combining can either be done by changing the injection currents $(0.02 \mathrm{~nm} / \mathrm{A})$ or more efficiently by changing the laser temperatures $(0.09 \mathrm{~nm} / \mathrm{K})$. Both the spectral characteristics and the tunability prove both lasers to be suitable for spectral beam combining with the specified grating.

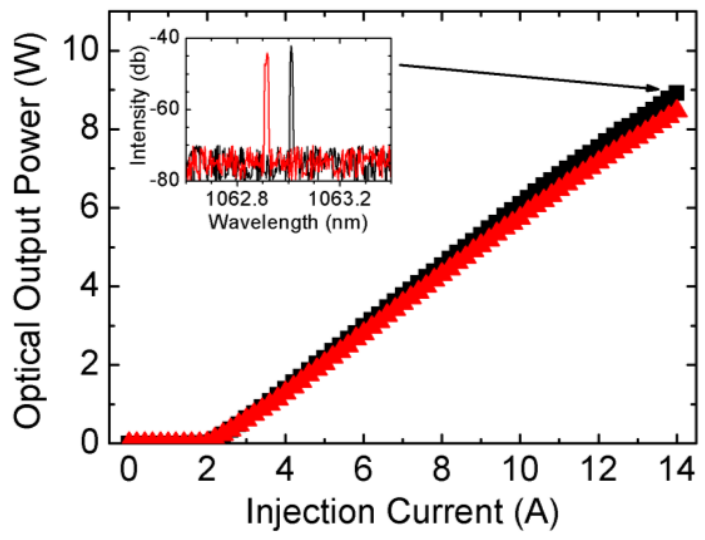

Fig. 2. Power current characteristics for both DBR-tapered diode lasers (Squares: transmitted laser, Triangles: diffracted laser). Both lasers are operated at $300 \mathrm{~mA}$ to the ridge waveguides and operating temperatures set to $20{ }^{\circ} \mathrm{C}$. The inset shows the corresponding spectra emitted at $14 \mathrm{~A}$ to the tapered amplifiers.

When properly adjusting the wavelengths of both lasers and the angle of incidence of the diffracted laser on the grating a combined output power of $16.08 \mathrm{~W}$ is achieved at maximum current (Fig. 3). At these settings the diffracted laser emits $8.05 \mathrm{~W}$ at $1063.17 \mathrm{~nm}$ and $23.0^{\circ} \mathrm{C}$. The transmitted laser emits $9.11 \mathrm{~W}$ at $1062.65 \mathrm{~nm}$ and 
$15.47{ }^{\circ} \mathrm{C}$. This corresponds to a conversion efficiency of $93.7 \%$ and an electro-optical efficiency of $25 \%$. At lower currents the combining efficiencies even increase up to $95.4 \%$. This could be explained with a slight degradation of laser parameters at higher currents. The general loss in spectral beam combing can be explained by a finite divergence of the laser beams and non-uniformities of the grating. The beam propagation parameters $\left(\mathrm{M}^{2}{ }_{1 / \mathrm{e}}{ }^{2}\right)$ of the combined beam at maximum current are measured to be 1.8 in the fast axis and 3.3 in the slow axis. These values are comparable to the values measured for the laser with lowest beam quality. A similar behavior is observed at low currents where the values are measured to be 1.6 in the fast axis and 2.4 in the slow axis.

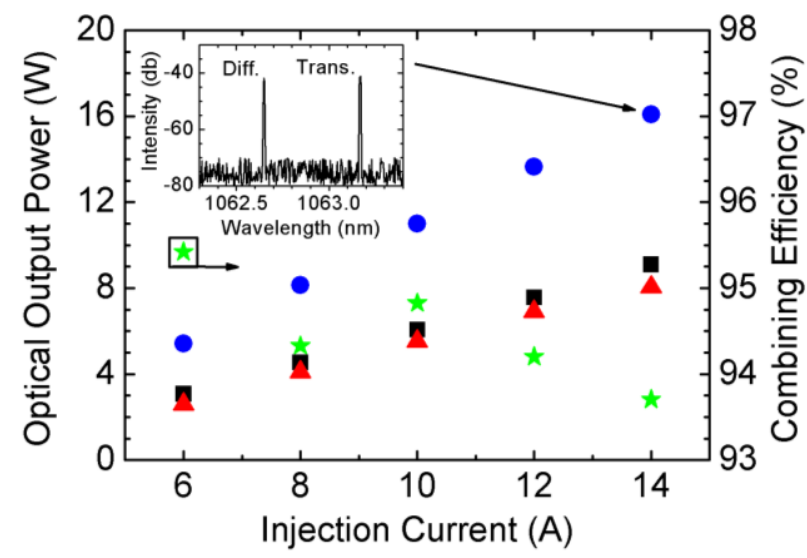

Fig. 3. Optical output power of the individual DBR-tapered diode lasers and the combined beam (Squares: transmitted laser, Triangles: diffracted laser, Circles: combined beam). The star symbols represent the corresponding combining efficiency. The inset shows the corresponding spectra emitted at maximum currents.

The fact that both beams can efficiently be combined and the wavelengths are separated enables subsequent sumfrequency generation, enhancing the green output power we achieved by frequency conversion of similar lasers so far [4]. In this context also the fact that the spectral characteristics as well as their beam propagation parameters are not affected is advantageous. We are currently working on these experiments and will show further results.

\section{Summary}

More than $16 \mathrm{~W}$ of output power are achieved by spectral beam combining of two $1063 \mathrm{~nm}$ DBR-tapered diode lasers. Using a reflecting volume Bragg grating we achieve combining efficiencies between $93 \%$ and $96 \%$. As expected the spectral characteristics as well as the beam propagation parameters are not affected by the grating. The resulting beam still shows single-mode emission with side-mode suppression $>25 \mathrm{~dB}$. The beam propagation parameters being 1.8 (fast axis) and 3.3 (slow axis) match the values of the tapered diode laser with the lowest beam quality.

This clearly shows the potential of power scaling using multiple high-power DBR-tapered diode lasers. Furthermore, the dimensions of the diode lasers themselves should enable efficient, cost-effective and compact laser systems emitting single near-diffraction limited beams with multiple tens of Watts. Additionally, the spectral separation of the combined beams could be used for sum-frequency generation.

\section{References}

[1] D. Vijayakumar, O. B. Jensen, R. Ostendorf, T. Westphalen, and B. Thestrup, "Spectral beam combining of a $980 \mathrm{~nm}$ tapered diode laser bar," Opt. Express 18, 893-898 (2010).

[2] O. Andrusyak, V. Smirnov, G. Venus, V. Rotar, and L. Glebov, "Spectral Combining and Coherent Coupling of Lasers by Volume Bragg Gratings," IEEE Sel. Top. Quantum Electron. 15, 344-353 (2009).

[3] B. Sumpf, K.-H. Hasler, P. Adamiec, F. Bugge, F. Dittmar, J. Fricke, H. Wenzel, M. Zorn, G. Erbert, and G. Tränkle, " High-Brightness Quantum Well Tapered Lasers," IEEE Sel. Top. Quantum Electron. 15, 1009-1020 (2009).

[4] O. B. Jensen, P. E. Andersen, B. Sumpf, K.-H. Hasler, G. Erbert, and P. M. Petersen, "1.5 W green light generation by single-pass second harmonic generation of a single-frequency tapered diode laser," Opt. Express 17, 6532-6539 (2009). 